\title{
On Weak Compactness and Lower Closure Results for Pettis Integrable (Multi)Functions
}

by

\author{
Erik J. BALDER and Anna Rita SAMBUCINI \\ Presented by Czestaw OLECH
}

Summary. In $[4,5,7]$ an abstract, versatile approach was given to sequential weak compactness and lower closure results for scalarly integrable functions and multifunctions. Its main tool is an abstract version of the Komlós theorem, which applies to scalarly integrable functions. Here it is shown that this same approach also applies to Pettis integrable multifunctions, because the abstract Komlós theorem can easily be extended so as to apply to generalized Pettis integrable functions. Some results in the literature are thus unified.

1. Introduction. In $[4,5]$ and subsequently [7] an abstract, versatile approach was given to sequential weak compactness and lower closure results for scalarly measurable functions and multifunctions. The main tool introduced there is an abstract Komlós theorem, which was obtained from Komlós's classical theorem [17] by means of a diagonal selection argument involving point-separation and compactness arguments. Subsequently, the same technique has been adopted by several authors who studied weak compactness and lower closure for Pettis integrable (multi)functions. Here we show that, instead of adopting the method of proof of [5], one can also directly apply the main results of that work. That is to say, we show that Theorem 2.1 and Corollary 2.2 of [5] apply directly to Pettis integrable (multi)functions, thanks to a well-known characterization of Pettis integrability $[19,21,22]$. In this way, some results in the literature are unified.

2000 Mathematics Subject Classification: Primary 28A20, $28 \mathrm{~B} 05$.

Key words and phrases: weak compactness, Pettis integrable functions, multifunctions, Komlós theorem.

Support from Istituto Nazionale di Alta Matematica (GNAMPA) enabled the first author to visit the University of Perugia in May-June 2002. 
2. An abstract Komlós theorem and its extension. In Theorem 2.1 of this section we recall the abstract Komlós theorem of $[4,5]$. This is followed by a presentation of a generalized notion of Pettis integrability and the extension of Theorem 2.1 to such functions (see Theorem 2.4 below). Let $(\Omega, \mathcal{F}, \mu)$ be a finite measure space and let $Y$ be a convex cone in the sense of [12] (i.e., $(Y,+)$ is an Abelian semigroup satisfying the usual laws of multiplication with nonnegative scalars), equipped with a suitable topology that is compatible with the operation + and with multiplication by scalars. In the applications presented here $Y$ will be just the hyperspace $\operatorname{cwk}(E)$, consisting of all nonempty convex and weakly compact subsets of a separable Banach space $E$, or it will be $E$ itself. Of course, then + is just set addition. Following [5], we shall say that a sequence $\left(f_{m}\right)$ of functions $f_{m}: \Omega \rightarrow Y K$-converges to a function $f_{0}: \Omega \rightarrow Y$ (notation: $f_{m} \stackrel{K}{\rightarrow} f_{0}$ ) if for every subsequence $\left(f_{m_{i}}\right)$ of $\left(f_{m}\right)$,

$$
\lim _{N \rightarrow \infty} \frac{1}{N} \sum_{i=1}^{N} f_{m_{i}}(\omega)=f_{0}(\omega) \quad \text { for a.e. } \omega \text { in } \Omega .
$$

Here the exceptional null set is allowed to depend on the particular subsequence $\left(f_{m_{i}}\right)$ (in fact, without this escape valve $K$-convergence would just amount to pointwise a.e. convergence on $\Omega$ ). The above $K$-convergence notion was inspired by a fundamental paper of Komlós [17]. From [5] we recall the abstract Komlós theorem (see also additional comments in [4] and [7]). Let $\mathcal{A}$ be a collection of $\mathcal{F} \times \mathcal{B}(Y)$-measurable functions $a: \Omega \times Y \rightarrow \mathbb{R}$ such that for every $\omega \in \Omega$,

$$
a(\omega, \cdot) \text { is affine and continuous on } Y \text {. }
$$

Following [5], a function $f: \Omega \rightarrow Y$ is called $\mathcal{A}$-scalarly measurable if the scalar function $a(\cdot, f(\cdot))$ is $\mathcal{F}$-measurable for every $a \in \mathcal{A}$. The collection $\mathcal{A}$ is supposed to have a countable subcollection $\mathcal{A}_{0}:=\left\{a_{j}: j \in \mathbb{N}\right\}$ with the following point-separating property: for every $\omega \in \Omega$ and for every $y, z \in Y$,

$$
a_{j}(\omega, y)=a_{j}(\omega, z) \text { for all } j \in \mathbb{N} \text { if and only if } y=z .
$$

Let $h: \Omega \times Y \rightarrow[0,+\infty]$ be a given function such that for every $\omega \in \Omega$,

$$
h(\omega, \cdot) \text { is convex and sequentially inf-compact on } Y \text {. }
$$

The latter means that for every $\omega \in \Omega$ and $\beta \in \mathbb{R}$ the set $\{y \in Y: h(\omega, y)$ $\leq \beta\}$ must be sequentially compact. Under the above assumptions the abstract Komlós theorem, obtained in [5, Theorem 2.1], states the following:

THEOREM 2.1 (abstract Komlós theorem). Let $\left(f_{n}\right)_{n \in \mathbb{N}}$ be a sequence of $\mathcal{A}$-scalarly measurable functions $f_{n}: \Omega \rightarrow Y$ such that

$$
\sup _{n} \int_{\Omega}\left|a_{j}\left(\omega, f_{n}(\omega)\right)\right| \mu(d \omega)<+\infty \quad \text { for every } j
$$


and

$$
\sigma:=\sup _{n} \int_{\Omega}^{*} h\left(\omega, f_{n}(\omega)\right) \mu(d \omega)<+\infty .
$$

Then there exist a subsequence $\left(f_{n_{j}}\right)$ of $\left(f_{n}\right)$ and an $\mathcal{A}$-scalarly measurable function $f_{*}: \Omega \rightarrow Y$ such that

(a) $f_{n_{j}} \stackrel{K}{\rightarrow} f_{*}$,

(b) $\int_{\Omega}^{*} h\left(\omega, f_{*}(\omega)\right) \mu(d \omega) \leq \sigma<+\infty$.

Here $\int_{\Omega}^{*}$ refers to outer integration with respect to the measure $\mu$. That is to say, for a possibly nonmeasurable function $\psi: \Omega \rightarrow[-\infty,+\infty]$ we define

$$
\int_{\Omega}^{*} \psi d \mu:=\inf \left\{\int_{\Omega} \phi d \mu: \phi \in \mathcal{L}_{\mathbb{R}}^{1}(\Omega, \mathcal{F}, \mu), \phi \geq \psi \text { a.e. }\right\},
$$

with the understanding that the infimum over the empty set equals $+\infty$. Corollary 2.2 of [5] contains the following lower semicontinuity result:

Corollary 2.2. In Theorem 2.1 the inequality

$$
\liminf _{m} \int_{\Omega} g\left(\omega, f_{m}(\omega)\right) \mu(d \omega) \geq \int_{\Omega} g\left(\omega, f_{*}(\omega)\right) \mu(d \omega)
$$

is valid for every $\mathcal{F} \times \mathcal{B}(Y)$-measurable $g: \Omega \times Y \rightarrow(-\infty,+\infty]$ such that $g(\omega, \cdot)$ is convex and lower semicontinous and $\left\{g^{-}\left(\cdot, f_{m}(\cdot)\right): m \in \mathbb{N}\right\}$ is uniformly integrable, where $g^{-}:=\max (0,-g)$.

The following addendum to (a) of Theorem 2.1 is elementary; here seq-cl $C$ stands for the set of all $y \in Y$ for which there is a sequence $\left(c_{k}\right) \subset C$ with $c_{k} \rightarrow y$.

Corollary 2.3. In Theorem 2.1,

(c) $f_{*}(\omega) \in \bigcap_{p=1}^{\infty} \operatorname{seq}-\operatorname{cl} \operatorname{co}\left\{f_{m}(\omega): m \geq p\right\}$ for a.e. $\omega$ in $\Omega$.

Let $\mathcal{A}_{1}$ be a given fixed subset of $\mathcal{A}$. Define an $\mathcal{A}$-scalarly measurable function $f: \Omega \rightarrow Y$ to be of class $U I\left(\mathcal{A}_{1}\right)$ if

$$
\left\{a(\cdot, f(\cdot)): a \in \mathcal{A}_{1}\right\} \text { is uniformly integrable. }
$$

In the next section we shall see that Pettis integrable (multi)functions are captured by this notion. The main result of this note is as follows. It is a straightforward consequence of Theorem 2.1 and the above two corollaries (conversely, it also implies them, as is seen by artificially including the null function 0 on $\Omega \times Y$ into $\mathcal{A}$ and setting $\left.\mathcal{A}_{1}:=\{0\}\right)$.

THEOREM 2.4. Let $\left(f_{n}\right)_{n \in \mathbb{N}}$ be a sequence of $\mathcal{A}$-scalarly measurable functions $f_{n}: \Omega \rightarrow Y$ of class $U I\left(\mathcal{A}_{1}\right)$ such that $(2.1)-(2.2)$ hold and

$$
\left\{a\left(\cdot, f_{n}(\cdot)\right): a \in \mathcal{A}_{1}, n \in \mathbb{N}\right\} \text { is uniformly integrable. }
$$


Then there exist a subsequence $\left(f_{n_{j}}\right)$ of $\left(f_{n}\right)$ and an $\mathcal{A}$-scalarly measurable function $f_{*}: \Omega \rightarrow Y$ of class $U I\left(\mathcal{A}_{1}\right)$ such that (a)-(b) of Theorem 2.1 and (c) of Corollary 2.3 hold, as well as

(d) $\lim _{j \rightarrow \infty} \int_{B} a\left(\omega, f_{n_{j}}(\omega)\right) \mu(d \omega)=\int_{B} a\left(\omega, f_{*}(\omega)\right) \mu(d \omega)$ for every $a \in \mathcal{A}_{1}$, $B \in \mathcal{F}$.

Proof. (a), (b), (c) follow as in Theorem 2.1 and Corollary 2.3, and (d) is obtained directly by applying Corollary 2.2 to both $g=a$ and $g=-a$, so only the fact that $f_{*}$ is of class $U I\left(\mathcal{A}_{1}\right)$ must be demonstrated. By the theorem of de la Vallée Poussin [10, II.22] there exists a convex, continuous nondecreasing function $h^{\prime}: \mathbb{R}_{+} \rightarrow \mathbb{R}_{+}$with $\lim _{\xi \rightarrow \infty} h^{\prime}(\xi) / \xi=+\infty$ such that

$$
\gamma:=\sup _{a \in \mathcal{A}_{1}, n \in \mathbb{N}} \int_{\Omega} h^{\prime}\left(\left|a\left(\omega, f_{n}(\omega)\right)\right|\right) \mu(d \omega)<+\infty .
$$

For every $a \in \mathcal{A}_{1}$ the formula $g(\omega, y):=h^{\prime}(|a(\omega, y)|)$ defines a function $g: \Omega \times Y \rightarrow \mathbb{R}_{+}$which clearly satisfies the conditions of Corollary 2.2 (in particular, here $g^{-}=0$ ). This gives

$$
\int_{\Omega} h^{\prime}\left(\left|a\left(\omega, f_{*}(\omega)\right)\right|\right) \mu(d \omega)=\int_{\Omega} g\left(\omega, f_{*}(\omega)\right) \mu(d \omega) \leq \gamma
$$

for every $a \in \mathcal{A}_{1}$. So by the reverse part of de la Vallée Poussin's theorem it follows that $\left\{\left|a\left(\cdot, f_{*}(\cdot)\right)\right|: a \in \mathcal{A}_{1}\right\}$ is uniformly integrable. Hence, $f_{*}$ is of class $U I\left(\mathcal{A}_{1}\right)$.

3. Applications to Pettis integration. In this section we shall apply Theorem 2.4 to Pettis integrable (multi)functions, very much in the same way as was done for Bochner integrable (multi)functions by Balder and Hess to obtain [7, Theorem 7.1] as a direct application of Theorem 2.1.

Let $E$ be a separable Banach space, whose dual is denoted by $E^{*}$. Let $\operatorname{cwk}(E)$ be the collection of all nonempty, convex and weakly compact subsets of $E$. A multifunction $F: \Omega \rightarrow \operatorname{cwk}(E)$ is called scalarly integrable if for every $x^{*} \in E^{*}$ the scalar function $s\left(x^{*} \mid F(\cdot)\right)$ is in $\mathcal{L}_{\mathbb{R}}^{1}(\Omega, \mathcal{F}, \mu)$. Here $s\left(x^{*} \mid C\right):=\sup _{x \in C}\left\langle x^{*}, x\right\rangle$ denotes the support function of $C \in \operatorname{cwk}(E)$. From now on $\operatorname{cwk}(E)$ will be equipped with the initial topology for the functionals $s\left(x^{*} \mid \cdot\right), x^{*} \in E^{*}$, i.e., the coarsest topology on $\operatorname{cwk}(E)$ for which those functionals are continuous. Recall from [11] that a multifunction $F: \Omega \rightarrow \operatorname{cwk}(E)$ is said to be Pettis integrable if it is scalarly integrable and if for every $B \in \mathcal{F}$ there exists $C_{B} \in \operatorname{cwk}(E)$ such that

$$
s\left(x^{*} \mid C_{B}\right)=\int_{B} s\left(x^{*} \mid F(\omega)\right) \mu(d \omega) \quad \text { for every } x^{*} \in E^{*} ;
$$

see Theorem 5.4 of [11] for a number of equivalent definitions. The following characterization of Pettis integrability is due to Ziat [21, Theorem 3.2] 
(see [22] for a corrected proof of this result and see [11] for much more general characterizations). This extends a well known Pettis integrability criterion for ordinary functions [19]. Below $U^{*}$ stands for the closed unit ball in $E^{*}$.

Proposition 3.1. For every scalarly integrable multifunction $F: \Omega \rightarrow$ $\operatorname{cwk}(E)$ the following are equivalent:

(i) $F$ is Pettis integrable,

(ii) the collection $\left\{s\left(x^{*} \mid F(\cdot)\right): x^{*} \in U^{*}\right\}$ is uniformly integrable.

In view of this criterion, we now have the following corollary of Theorem 2.4:

TheOREM 3.2. Let $\left(F_{n}\right)$ be a sequence of Pettis integrable multifunctions $F_{n}: \Omega \rightarrow \operatorname{cwk}(E)$ such that there exists $h: \Omega \times \operatorname{cwk}(E) \rightarrow[0,+\infty]$ with

$$
\sup _{n} \int_{\Omega}^{*} h\left(\omega, F_{n}(\omega)\right) \mu(d \omega)<+\infty
$$

$h(\omega, \cdot)$ is convex and inf-compact on $\operatorname{cwk}(E)$ for a.e. $\omega$ in $\Omega$,

and such that

$$
\left\{s\left(x^{*} \mid F_{n}(\cdot)\right): x^{*} \in U^{*}, n \in \mathbb{N}\right\} \text { is uniformly integrable. }
$$

Then there exist a subsequence $\left(F_{n_{j}}\right)$ of $\left(F_{n}\right)$ and a Pettis integrable $F_{*}$ : $\Omega \rightarrow \operatorname{cwk}(E)$ such that

(a') $F_{n_{j}} \stackrel{K}{\rightarrow} F_{*}$,

$\left(\mathrm{b}^{\prime}\right) \int_{\Omega}^{*} h\left(\omega, F_{*}(\omega)\right) \mu(d \omega)<+\infty$,

(c') $F_{*}(\omega) \subset \bigcap_{p=1}^{\infty} \operatorname{clco\bigcup } \bigcup_{j \geq p} F_{n_{j}}(\omega)$ for a.e. $\omega$ in $\Omega$,

$\left(\mathrm{d}^{\prime}\right) \lim _{j \rightarrow \infty} \int_{B} s\left(x^{*} \mid F_{n_{j}}(\omega)\right) \mu(d \omega)=\int_{B} s\left(x^{*} \mid F_{*}(\omega)\right) \mu(d \omega)$ for every $x^{*} \in E^{*}$ and $B \in \mathcal{F}$.

Proof. Let $Y:=\operatorname{cwk}(E)$. For $x^{*} \in E^{*}$ define $a_{x^{*}}(\omega, C):=s\left(x^{*} \mid C\right)$. Let $\mathcal{A}:=\left\{a_{x^{*}}: x^{*} \in E^{*}\right\}$ and $\mathcal{A}_{1}:=\left\{a_{x^{*}}: x^{*} \in U^{*}\right\}$. Then $\mathcal{A}$-scalar measurability is just scalar measurability as defined above, and by Proposition 3.1 a scalarly measurable $F: \Omega \rightarrow \operatorname{cwk}(E)$ is of class $U I\left(\mathcal{A}_{1}\right)$ if and only if it is Pettis integrable. Also, it is well known that there exists a countable, Mackey dense subset $\left(x_{j}^{*}\right)$ of $E^{*}$ that separates the sets in $\operatorname{cwk}(E)$ [16]. Form $\mathcal{A}_{0}:=\left\{a_{x_{j}^{*}}: j \in \mathbb{N}\right\}$ to obtain a point-separating collection of affine, continuous functions on $Y=\operatorname{cwk}(E)$. After this one can apply Theorem 2.4, which gives $\left(\mathrm{a}^{\prime}\right),\left(\mathrm{b}^{\prime}\right)$ and $\left(\mathrm{d}^{\prime}\right)$ immediately. To obtain $\left(\mathrm{c}^{\prime}\right)$ from $(\mathrm{c})$, one applies the following basic facts: (1) for any collection $\left\{C_{i}: i \in I\right\}$ in $\operatorname{cwk}(E)$ each element of the convex hull co $\left\{C_{i}: i \in C_{i}\right\}$ (i.e., the convex hull taken in the cone $\operatorname{cwk}(E))$ is a set that is contained in the subset $\operatorname{co}\left(\bigcup_{i \in I} C_{i}\right)$ of $E$, 
(2) if a sequence $\left(C_{k}\right)$ in $\operatorname{cwk}(E)$ scalarly converges to $C \in \operatorname{cwk}(E)$ (i.e., $\lim _{k} s\left(x^{*} \mid C_{k}\right)=s\left(x^{*} \mid C\right)$ for every $x^{*} \in E^{*}$ ), then $C \subset \operatorname{clco\bigcup } \bigcup_{k} C_{k}$ (apply the Hahn-Banach theorem).

Corollary 3.3. Let $\left(F_{n}\right)$ be a sequence of Pettis integrable multifunctions $F_{n}: \Omega \rightarrow \operatorname{cwk}(E)$ such that there exists $\Gamma: \Omega \rightarrow \operatorname{cwk}(E)$ with

and such that

$$
\bigcup_{n} F_{n}(\omega) \subset \Gamma(\omega) \quad \text { for a.e. } \omega \text { in } \Omega
$$

$$
\left\{s\left(x^{*} \mid F_{n}(\cdot)\right): x^{*} \in U^{*}, n \in \mathbb{N}\right\} \text { is uniformly integrable. }
$$

Then $\left(\mathrm{a}^{\prime}\right)$, $\left(\mathrm{c}^{\prime}\right)$ and $\left(\mathrm{d}^{\prime}\right)$ of Theorem 3.2 hold. Moreover, $\left(\mathrm{c}^{\prime}\right)$ then amounts to

$\left(\mathrm{c}^{\prime \prime}\right) F_{*}(\omega) \subset \mathrm{clcow}-\mathrm{Ls} F_{n}(\omega)$ for a.e. $\omega$ in $\Omega$.

Recall here from [14] that for any sequence $\left(C_{n}\right)$ of sets $C_{n} \subset E$ the $w$-sequential upper limit (alias $w$-limes superior in the sense of Kuratowski), denoted by $w$-Ls $C_{n}$, is defined as the collection of all points $x \in E$ for which there exist a subsequence $\left(C_{n_{j}}\right)$ and associated points $x_{n_{j}} \in C_{n_{j}}$ with $x=\lim _{j} x_{n_{j}}$.

Proof. Define $h(\omega, C):=0$ if $C \subset \Gamma(\omega)$ and $h(\omega, C):=+\infty$ otherwise. Then the convexity of $h(\omega, \cdot)$ is immediate and its inf-compactness follows by Blaschke-type results, such as [7, Lemma 3.3] or [13, Lemma 5.1]). The equivalence of $\left(\mathrm{c}^{\prime}\right)$ and $\left(\mathrm{c}^{\prime \prime}\right)$, given the condition involving $\Gamma$, follows by Lemma 3.3 of [7] (see [14] for the original result).

This corollary generalizes Theorems 4.1 and 4.2 of [21]. Part $\left(\mathrm{d}^{\prime}\right)$ generalizes Theorem 2.6 of [8]. Recall that a function $f: \Omega \rightarrow E$ is said to be scalarly integrable (respectively Pettis integrable) if the corresponding $\operatorname{cwk}(E)$-valued multifunction $\omega \mapsto\{f(\omega)\}$ is scalarly integrable (respectively Pettis integrable) [19].

TheOREM 3.4. Let $\left(f_{n}\right)$ be a sequence of Pettis integrable functions $f_{n}$ : $\Omega \rightarrow E$ such that there exists $h: \Omega \times E \rightarrow[0,+\infty]$ with

$$
\sup _{n} \int_{\Omega}^{*} h\left(\omega, f_{n}(\omega)\right) \mu(d \omega)<+\infty
$$

$h(\omega, \cdot)$ is convex and inf-compact on $E$ for a.e. $\omega$,

and such that

$$
\left.\left\{\left\langle x^{*}, f_{n}(\cdot)\right)\right\rangle: x^{*} \in U^{*}, n \in \mathbb{N}\right\} \text { is uniformly integrable. }
$$

Then there exist a subsequence $\left(f_{n_{j}}\right)$ of $\left(f_{n}\right)$ and a Pettis integrable $f_{*}$ : $\Omega \rightarrow E$ such that 


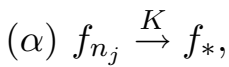

( $\beta) \int_{\Omega}^{*} h\left(\omega, f_{*}(\omega)\right) \mu(d \omega)<+\infty$,

$(\gamma) f_{*}(\omega) \in \bigcap_{p=1}^{\infty} \operatorname{clco\bigcup } \bigcup_{j \geq p} f_{n_{j}}(\omega)$ for a.e. $\omega$ in $\Omega$,

$(\delta) \lim _{j \rightarrow \infty} \int_{B}\left\langle x^{*}, f_{n_{j}}(\omega)\right\rangle \mu(d \omega)=\int_{B}\left\langle x^{*}, f_{*}(\omega)\right\rangle \mu(d \omega)$ for every $x^{*} \in E^{*}$ and $B \in \mathcal{F}$.

For $Y:=E$ instead of $Y=\operatorname{cwk}(E)$ the proof is an obvious analogue of the proof of Theorem 3.2 by means of Theorem 2.4. Note that there is no need to formulate an analogue for functions of Corollary 3.3, since this is already contained in the latter (specialize to single-valued multifunctions).

Until now, we supposed that $E$ was a separable Banach space. For a quasi-complete locally convex Suslin vector space $\widetilde{E}$ the above definition of Pettis integrability of a function $f: \Omega \rightarrow \widetilde{E}$ can be repeated verbatim (recall that a Hausdorff topological space is Suslin if it is the continuous surjective image of a Polish space). In this more general setting the criterion contained in Proposition 3.1 has a version for functions (we do not know of a similar counterpart for multifunctions). The following result comes from [18, Theorem 1]:

Proposition 3.5. Let $f: \Omega \rightarrow \widetilde{E}$ be scalarly integrable such that

(a) for every equicontinuous set $K \subset \widetilde{E}^{*}$ the collection $\left\{\left\langle x^{*}, f\right\rangle: x^{*} \in K\right\}$ is uniformly integrable,

(b) there exists a sequence $\left(f_{n}\right)$ of Pettis integrable functions $f_{n}: \Omega \rightarrow \widetilde{E}$ such that

$\lim _{n} \int_{B}\left\langle x^{*}, f_{n}\right\rangle d \mu=\int_{B}\left\langle x^{*}, f\right\rangle d \mu \quad$ for every $x^{*} \in \widetilde{E}^{*}$ and every $B \in \mathcal{F}$. Then $f$ is Pettis integrable.

We now have the following extension of Theorem 3.4 for functions taking values in the quasi-complete locally convex Suslin vector space $\widetilde{E}$ :

ThEOREM 3.6. Let $\left(f_{n}\right)$ be a sequence of Pettis integrable functions $f_{n}$ : $\Omega \rightarrow \widetilde{E}$ such that (3.3)-(3.4) hold (for $\widetilde{E}$ instead of $E$ ) and such that for every equicontinuous $K \subset \widetilde{E}^{*}$,

$$
\left\{\left\langle x^{*}, f_{n}(\cdot)\right\rangle: x^{*} \in K, n \in \mathbb{N}\right\} \text { is uniformly integrable. }
$$

Then there exist a subsequence $\left(f_{n_{j}}\right)$ of $\left(f_{n}\right)$ and a Pettis integrable $f_{*}$ : $\Omega \rightarrow \widetilde{E}$ such that

$$
\begin{aligned}
& \left(\alpha^{\prime}\right) f_{n_{j}} \stackrel{K}{\rightarrow} f_{*}, \\
& \left(\beta^{\prime}\right) \int_{\Omega}^{*} h\left(\omega, f_{*}(\omega)\right) \mu(d \omega)<+\infty,
\end{aligned}
$$


$\left(\gamma^{\prime}\right) f_{*}(\omega) \in \bigcap_{p=1}^{\infty} \operatorname{clco\bigcup } \bigcup_{j \geq p} f_{n_{j}}(\omega)$ for a.e. $\omega$ in $\Omega$,

$\left(\delta^{\prime}\right) \lim _{j \rightarrow \infty} \int_{B}\left\langle x^{*}, f_{n_{j}}(\omega)\right\rangle \mu(d \omega)=\int_{B}\left\langle x^{*}, f_{*}(\omega)\right\rangle \mu(d \omega)$ for every $x^{*} \in E^{*}$ and $B \in \mathcal{F}$.

Proof. Let $Y:=\widetilde{E}$. For $x^{*} \in \widetilde{E}^{*}$ we set $a_{x^{*}}(\omega, x):=\left\langle x^{*}, x\right\rangle$. Let $\mathcal{A}:=\left\{a_{x^{*}}: x^{*} \in E^{*}\right\}$; then, clearly, $\mathcal{A}$-scalar measurability is ordinary scalar measurability. By [9, III.32] there exists a countable Mackey dense subset $\left(x_{j}^{*}\right)$ of $\widetilde{E}^{*}$ that separates the points of $\widetilde{E}$. Setting $\mathcal{A}_{0}:=\left\{a_{x_{j}^{*}}: j \in \mathbb{N}\right\}$, we can now apply Theorem 3.2. This gives $\left(\alpha^{\prime}\right),\left(\beta^{\prime}\right),\left(\gamma^{\prime}\right)$ and $\left(\delta^{\prime}\right)$, so it only remains to prove that $f_{*}$ is Pettis integrable. We do this by invoking Proposition 3.5. For any equicontinuous set $K \subset \widetilde{E}^{*}$ the proof of Theorem 2.4 can be repeated, with $\left\{a_{x^{*}}: x^{*} \in K\right\}$ in the role of $\mathcal{A}_{1}$. This gives that $\left\{\left\langle x^{*}, f\right\rangle: x^{*} \in K\right\}$ is uniformly integrable. Hence condition (a) of Proposition 3.5 holds. Let $x^{*} \in E^{*}$. By the uniform integrability of $\left.\left\{\left\langle x^{*}, f_{n}(\cdot)\right)\right\rangle: n \in \mathbb{N}\right\}$ it follows from applying Corollary 2.2 two times $\left(\operatorname{set} g(\omega, x):=1_{B}(\omega)\left\langle x^{*}, x\right\rangle\right.$ and $g(\omega, x):=-1_{B}(\omega)\left\langle x^{*}, x\right\rangle$ ) that $\lim _{j} \int_{B}\left\langle x^{*}, f_{n_{j}}\right\rangle d \mu=\int_{B}\left\langle x^{*}, f_{*}\right\rangle d \mu$ for every $B \in \mathcal{F}$. Hence, also condition (b) of Proposition 3.5 holds.

This generalizes the first part of Theorem 3.4 in [1]; the second part of that result is a Fatou-type lemma. Such Fatou-type results can also be given in the context of this section and the previous one. However, Fatou-type results that strictly apply to Pettis integrable (multi)functions (as opposed to those for Bochner integrable (multi)functions $[3,6,7,20]$ ) are difficult to formulate, for somehow they must involve uniform $L^{1}$-bounds. Recent results on this subject can be found in [15], where a countable partition figures, on each of whose parts a Bochner Fatou-type result from [7, Theorem 2.5] is applied.

Acknowledgments. We are grateful to an anonymous referee who made several helpful suggestions, and to Christian Hess (Paris) for sending us a copy of [15] after this paper was completed.

\section{References}

[1] A. Amrani, Lemme de Fatou pour l'intégrale de Pettis, Publ. Mat. 42 (1998), 67-79.

[2] A. Amrani and C. Castaing, Weak compactness in Pettis integration, Bull. Polish Acad. Sci. Math. 45 (1997), 139-150.

[3] E. J. Balder, Fatou's lemma in infinite dimensions, J. Math. Anal. Appl. 136 (1988), 450-465.

[4] -, Unusual applications of a.e. convergence, in: Almost Everywhere Convergence, G. A. Edgar and L. Sucheston (eds.), Academic Press, New York, 1989, 31-53.

[5] - New sequential compactness results for spaces of scalarly integrable functions, J. Math. Anal. Appl. 151 (1990), 1-16. 
[6] E. J. Balder and C. Hess, Fatou's lemma for multifunctions with unbounded values, Math. Oper. Res. 20 (1995), 175-188.

[7] —, - Two generalizations of Komlós' theorem with lower closure-type applications, J. Convex Anal. 3 (1996), 25-44.

[8] C. Castaing, Weak compactness and convergences in Bochner and Pettis integration, Vietnam J. Math. 24 (1996), 1-40.

[9] C. Castaing and M. Valadier, Convex Analysis and Measurable Multifunctions, Lecture Notes in Math. 580, Springer, Berlin, 1977.

[10] C. Dellacherie et P.-A. Meyer, Probabilités et Potentiel, Hermann, Paris, 1975.

[11] K. El Amri and C. Hess, On the Pettis integral of closed valued multifunctions, Set-Valued Anal. 8 (2000), 329-360.

[12] B. Fuchssteiner and W. Lusky, Convex Cones, North-Holland, Amsterdam 1981.

[13] C. Hess, On multivalued martingales whose values may be unbounded: martingale selectors and Mosco convergence, J. Multivariate Anal. 39 (1991), 175-201.

[14] - Measurability and integrability of the weak upper limit of a sequence of multifunctions, J. Math. Anal. Appl. 153 (1990), 226-249.

[15] C. Hess and H. Ziat, Théorème de Komlós pour des multifonctions intégrables au sens de Pettis et applications, Ann. Sci. Math. Québec, to appear.

[16] V. Klee and C. Olech, Characterizations of a class of sets, Math. Scand. 20 (1967), 290-296.

[17] J. Komlós, A generalization of a problem of Steinhaus, Acta Math. Acad. Sci. Hungar. 18 (1967), 217-229.

[18] K. Musiał, Vitali and Lebesgue convergence theorems for Pettis integral in locally convex spaces, Atti Sem. Mat. Fis. Univ. Modena 35 (1987), 159-165.

[19] - Topics in the theory of Pettis integration, Rend. Ist. Mat. Univ. Trieste 23 (1991), 177-262.

[20] N. C. Yannelis, Fatou's lemma in infinite dimensional spaces, Proc. Amer. Math. Soc. 102 (1988), 303-310.

[21] H. Ziat, Convergence theorems for Pettis integrable multifunctions, Bull. Polish Acad. Sci. Math. 45 (1997), 123-137.

[22] - On a characterization of Pettis integrable multifunctions, ibid. 48 (2000), $228-230$.

Erik J. Balder

Mathematical Institute

University of Utrecht, Budapestlaan 6

P.O. Box 80.010

3508 TA Utrecht, The Netherlands

E-mail: balder@math.uu.nl
Anna Rita Sambucini

Department of Mathematics and Informatics University of Perugia Via L. Vanvitelli 1 06100 Perugia, Italy E-mail: matears1@unipg.it

Received January 20, 2003;

received in final form June 20, 2003 